\title{
Once-yearly zoledronic acid in hip fracture prevention
}

\section{Oddom Demontiero Gustavo Duque}

Aging Bone Research Program, Nepean Clinical School, University of Sydney, Penrith, NSW, Australia; Department of Geriatric Medicine, Nepean Hospital, Penrith, NSW, Australia
Correspondence: Gustavo Duque Nepean Clinical School, Level 5, South Block, Nepean Hospital,

Penrith, NSW, Australia 2750

Tel +6I 247344279

Fax +6I 247341817

Email gduque@med.usyd.edu.au

\begin{abstract}
Osteoporosis is an escalating global problem. Hip fractures, the most catastrophic complication of osteoporosis, continue to cause significant mortality and morbidity despite increasing availability of effective preventative agents. Among these agents, oral bisphosphonates have been the first choice for the treatment and prevention of osteoporotic fractures. However, the use of oral bisphosphonates, especially in the older population, has been limited by their side effects and method of administration thus compromising their persistent use. The resultant low adherence by patients has undermined their full potential and has been associated with an increase in the incidence of fragility fractures. Recently, annual intravenous zoledronic acid (ZOL) has been approved for osteoporosis. Randomized controlled trials have demonstrated ZOL to be safe, have good tolerability and produce significant effect on bone mass and microarchitecture. Adherence has also been shown to be better with ZOL. Furthermore two large trials firmly demonstrated significant anti-osteoporotic effect $(\sim 59 \%$ relative risk reduction of hip fractures) and mortality benefit ( $28 \%$ reduction in mortality) of ZOL in older persons with recent hip fractures. In this review, we report the current evidence on the use of ZOL for the prevention of hip fractures in the elderly. We also report the pharmacological characteristics and the advantages and disadvantages of ZOL in this particular group.
\end{abstract}

Keywords: osteoporosis, zoledronic acid, hip fracture, elderly

\section{Introduction}

Osteoporosis is a disease associated with low bone mass, which predisposes to fractures. ${ }^{1,2}$ Osteoporosis is also a major public health problem that represents a significant burden for health care budgets due to the increasing prevalence of fractures and disability. ${ }^{3}$ Aging is the most important risk factor for osteoporosis ${ }^{4,5}$ being highly prevalent in both elderly men and women with its incidence expected to increase in the upcoming years. ${ }^{6,7}$

Although all fractures not associated with trauma in older adults should be interpreted as osteoporotic fractures, ${ }^{8}$ hip fractures represent the most typical and catastrophic events associated with osteoporosis in older adults. In fact, hip fractures are considered a predictor of mortality in both institutionalized and ambulatory older persons. With hip fractures, most deaths occur in the first 3 to 6 months following the event, of which $20 \%$ to $30 \%$ are causally related to the fracture event itself. ${ }^{9}$ Women with hip fractures have mortality rates $10 \%$ to $20 \%$ greater than expected for their age within the first year ${ }^{10}$ and this excess mortality persists for several years after the hip fracture. ${ }^{11}$ In fact, a white woman when sustaining a hip fracture at age 70 has an excess mortality of $3 \%, 4 \%, 7 \%$ and $13 \%$ at $1,2,5$ and 10 years after injury, respectively. ${ }^{11}$

Unfortunately, a significant proportion of elderly patients who have suffered a hip fracture do not receive further treatment for osteoporosis. ${ }^{12-17}$ Considering that the occurrence of a hip fracture is a predictor of new fractures, ${ }^{18-21}$ mortality ${ }^{9,22-24}$ and disability, ${ }^{25}$ a more pro-active and effective therapeutic approach should be encouraged in all elderly patients after presenting with hip fractures. 
There have been additional therapeutic agents available in the management of osteoporosis in recent years. ${ }^{26-28}$ New medications have shown to be effective for primary and secondary prevention of osteoporotic fractures. ${ }^{27,28} \mathrm{In}$ addition, less frequent dosing of bisphosphonates, a group of medications that constitute the mainstay of treatment for osteoporosis, have demonstrated their impact by improving compliance, a major limitation for effective osteoporosis treatment in the older population. ${ }^{28}$

Amongst the bisphosphonates, an annual dose of zoledronic acid (ZOL) has been recently proven as an effective approach for the management of osteoporosis. . $^{29,30}$ This intravenous dose has several advantages over other bisphosphonates, compliance being the most important. In this review, we will summarize the pharmacological characteristics of ZOL and the recent evidence on annual ZOL in primary and secondary prevention of hip fractures focusing on the evidence in the older population.

\section{Mechanisms of action of zoledronic acid Pharmacodynamics}

Bisphosphonates are pyrophosphate analogs with high affinity for hydroxyapatite and bind directly to mineralized bone. ${ }^{26,31}$ The bound bisphosphonate blocks the surface of bone and prevents osteoclasts from binding to the resorption surface. The bisphosphonates also inhibit osteoclastic activity, reduce the lifespan of the osteoclasts and alters the bone or bone mineral to reduce the rate of dissolution. ${ }^{32}$

There are two classes of bisphosphonates: the second generation that contains nitrogen in either an alkyl chain (alendronate, pamidronate and ibandronate) or within a heterocyclic ring (risedronate and ZOL) and the earlier generation that does not (etidronate and clodronate). Those without the nitrogen side chain have low antiresorptive potency and works by being incorporated into molecules of adenosine triphosphate (ATP). Subsequent intracellular accumulation of these nonhydrolyzable ATP analogues inhibit ATP-dependent cellular processes, thus inducing osteoclast-apoptosis. ${ }^{26,33,34}$ The more potent nitrogen containing bisphosphonates affect osteoclast activity and survival through the inhibition of the enzymes within the mevalonate pathway, the biosynthetic pathway for cholesterol and isoprenoid lipids such as farnesyl pyrophosphate (FPP) and geranylgeranyl diphosphate (GGPP). The key enzyme is thought to be FPP synthase. ${ }^{36}$ Biochemical studies ${ }^{31,36-38}$ have demonstrated that ZOL and other nitrogen-containing bisphosphonates inhibit FPP synthase, thus preventing the synthesis of FPP and GGPP, which are necessary for prenylation of small guanosine triphosphate (GTP)-binding proteins in osteoclasts. The loss of these prenylated proteins is associated with decreased osteoclast activity and apoptosis. ${ }^{39,40}$

The mechanism of apoptosis in osteoclasts was further studied by Benford et al..$^{39}$ Osteoclast apoptosis was associated with activation of caspase-3-like proteases, loss of mitochondrial membrane potential and classic morphologic changes. Of the bisphosphonates studied (risedronate, pamidronate, clodronate, etidronate, tiludronate, alendronate, and ZOL), ZOL was the most potent inhibitor of FPP synthase ${ }^{41}$ and caused the greatest increase in caspase-3-like activity at 48 hours after treatment. This order of potency in inhibiting FPP synthase $(\mathrm{ZOL}>$ risedronate $>$ ibandronate $>$ alendronate) (Figure 1) closely matched the order of antiresorptive potency. ${ }^{10}$ Ultimately the relative potency of different bisphosphonates is related to their binding affinities for hydroxyapatite and their degree of inhibition of FPP synthase. Nancollas et $a^{42}$ demonstrated that the different binding affinities of various bisphosphonates correlated with the differences in the $\mathrm{R} 2$ side chain $(\mathrm{ZOL}>$ alendronate $>$ ibandronate $=$ risedronate) (Figure 1$)$.

In addition, ZOL may have direct effects on osteoblasts. Studies examining its effects on BMD, bone architecture, bone strength and biochemical markers of bone metabolism have demonstrated favorable effects which included dosedependent prevention of increased cortical bone porosity and decreased bone resorption. ${ }^{43,44}$ In vitro studies also showed that ZOL inhibits vitamin D3-induced calcium release and that at effective doses for inhibiting bone resorption, it did not inhibit bone mineralization to any greater degree compared to other bisphosphonates..$^{38}$

\section{Pharmacokinetics}

$\mathrm{ZOL}$ is administered as a once-yearly 5 -mg intravenous infusion over no less than 15 minutes. ${ }^{45} \mathrm{ZOL}$ is not metabolized in humans and about $40 \%$ of an IV dose of ZOL is eliminated unchanged via the kidney. ${ }^{46}$ The remaining $60 \%$ binds to bone. In vivo studies in rats showed that high levels of 14C-labeled ZOL ( $>1.105 \mathrm{pmol} / \mathrm{g}$ ) distributed widely throughout the axial and appendicular skeleton, mandible, skull, and kidney within 1 hour after IV injection. ${ }^{38}$ Bone concentrations remained high although levels of ZOL in blood and soft tissues declined to low levels $(<1 \mathrm{pmol} / \mathrm{g})$ after a period of 8 months following administration.

A pharmacokinetic study ${ }^{47}$ showed that ZOL produced dose-proportional plasma concentrations that declined rapidly in a triphasic manner: rapid biphasic disappearance 

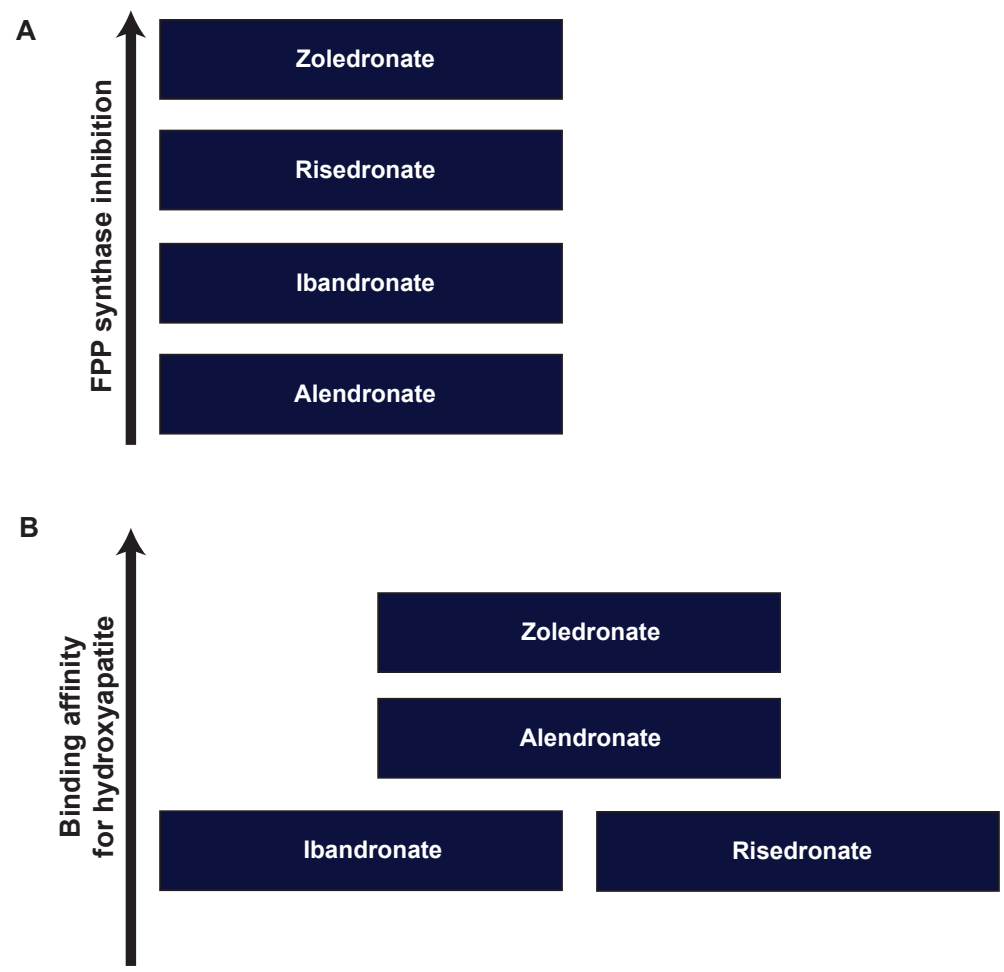

Figure I Compared with other bisphosphonates of the same generation, zoledronic acid has shown a higher inhibitory effect on farnesyl pyrophosphate synthase (FPP) (A) as well as higher affinity for hydroxyapatite in bones (B).

from the systemic circulation followed by a long elimination phase with a terminal elimination half-life of 146 hours. Twenty-four hours after injection, plasma concentrations declined to $<1 \%$ of the peak concentration at the end of infusion.

The substance is thought to have little or no capacity as a direct acting and/or irreversible metabolism dependent inhibitor of cytochrome P450 enzymes, and thus is unlikely to reduce the metabolic clearance of substances that are metabolized via the cytochrome P450 enzyme systems. No specific drug-drug interactions have been documented with ZOL.

The renal clearance of ZOL correlated with creatinine clearance. Small observed increases in AUC (0-24 hours), by about $30 \%$ to $40 \%$ in mild to moderate renal impairment, compared to a patient with normal renal function, and lack of accumulation of drug with multiple doses irrespective of renal function, suggest that dose adjustments of ZOL in mild (Clcr $=50-80$ $\mathrm{mL} / \mathrm{minute})$ and moderate $(\mathrm{Clcr}=35-50 \mathrm{~mL} / \mathrm{minute})$ renal impairment are not necessary. In fact, a recent study by Boonen et $\mathrm{al}^{48}$ looking at renal safety of annual ZOL infusions in 7714 osteoporotic postmenopausal women found that transient changes in renal function can occur following an annual ZOL infusion but, in the long term, renal function was not different from that in control patients. Nevertheless, as with other bisphosphonates, the use of ZOL in elderly patients with creatinine clearance $<35 \mathrm{~mL} /$ minute is not recommended due to limited clinical safety data in such patients.

\section{Safety and tolerability}

Overall ZOL is safe and well tolerated when used in the treatment and prevention of osteoporosis. The most common adverse events observed were acute phase reactions, ${ }^{29}$ usually characterized by flu-like symptoms, headache, bone pain, arthralgias and myalgias. Most of these symptoms occurred within the first 3 days after infusion and tended to resolve within several days after administration. They are usually easily managed with acetaminophen or ibuprofen and the incidence of these symptoms decreased with subsequent annual infusions.

\section{Renal effects}

No clinically significant long-term effects of ZOL on renal function have been observed so far. In the HORIZON-PFT, there was a significant increase in serum creatinine of more than $44 \mu \mathrm{mol} / \mathrm{L}$ in $1.3 \%$ of ZOL-treated patients, compared with $0.4 \%$ of placebo patients at 9 to 11 days post infusion. Within 30 days, however, the levels in more than $85 \%$ of 
patients had returned to within $44 \mu \mathrm{mol} / \mathrm{L}$ of pre-infusion values, and the remainder had returned to this level by the next annual follow-up. At 3 years, there was no significant difference in either serum creatinine levels or creatinine clearance between the placebo group and ZOL group. Boonen et $\mathrm{al}^{48}$ reported these changes testing the renal-function of 5035 osteoporotic postmenopausal women from the HORIZON Pivotal Fracture Trial. They also found that two of the four patients in the ZOL group who suffered acute renal failure requiring hospitalization had baseline creatinine clearance of $31 \mathrm{~mL}$ per min. Overall, they reported the mean decrease in serum creatinine in these elderly patients to be similar in both groups, as well as the overall number of observed renal adverse effects.

Comparable results were reported in HORIZON-RFT; ${ }^{30}$ the incidence of serum creatinine elevations greater than $44 \mu \mathrm{mol} / \mathrm{L}$ did not differ between the ZOL and placebo groups $(6.2 \%$ and $5.6 \%)$. The result from McClung et $\mathrm{al}^{49}$ trial is similar. Only $0.02 \%$ of patients in the ZOL group experienced a rise in serum creatinine or creatinine clearance. Furthermore these patients had low baseline creatinine clearance of around $30 \mathrm{~mL} / \mathrm{min}$. At the end of 1 year the renal function recovered to near baseline. Reid et $\mathrm{al}^{50}$ administering various doses of ZOL also did not observe any significant renal side effects.

\section{Atrial fibrillation (AF)}

The association with AF remains uncertain, as a causal relationship has not been established. In HORIZON-PFT, ${ }^{29}$ the number of patients who had arrhythmia in the ZOL group (266 patients, or 6.9\%) was significantly higher than that in the placebo group (203 patients, or 5.3\%; $\mathrm{p}=0.003$ ). The overall incidence of all AF adverse events was $2.4 \%$ of patients (94 of 3862) in the ZOL group, compared with $1.9 \%$ of patients ( 73 of 3852 ) in the placebo group. AF judged to be serious occurred in 50 patients in the ZOL group (1.3\%), as compared with 17 patients $(0.4 \%)$ in the placebo group. Among the 50 patients, the events occurred more than 30 days after infusion in 47 patients. Among 559 patients who underwent electrocardiography, the prevalence of ZOL (2.1\% in the ZOL group and $2.8 \%$ in the placebo group) and other electrocardiographic abnormalities did not differ significantly between the study groups. Interestingly the HORIZON-RFT ${ }^{30}$ did not find a difference in the incidence of AF events: 29 (2.8\%) in the ZOL group versus $27(2.6 \%)$ cases for the placebo group. In summary, data on the incidence of AF reported in clinical trials using $\mathrm{ZOL}$ for fracture prevention is limited to only two studies. More evidence is required to consider whether this finding is just chance or a real side effect that should be seriously considered when treating old patients.

\section{Osteonecrosis of the jaw (ONJ)}

The incidence of ONJ in patients treated for osteoporosis using bisphosphonates is estimated to be less than 1 in 100,000 patients. ${ }^{51}$ Although there have been case reports of ONJ in patients on alendronate ${ }^{51,52}$ and risedronate, ${ }^{53}$ there have been no reports of spontaneous ONJ in patents treated with ZOL. However, in HORIZON-PFT ${ }^{29}$ an independent adjudication committee blinded to study treatment identified two potential cases of ONJ, one in the placebo arm and one in the ZOL group.

\section{Other adverse effects}

Comparable number of patients in HORIZON-PFT ${ }^{29}$ and in HORIZON-RFT ${ }^{30}$ had notable declines of serum calcium levels (to $<7.5 \mathrm{mg} / \mathrm{dL}$ ) after administration of ZOL; but no symptomatic cases of hypocalcemia were observed. It is possible that the loading of vitamin $\mathrm{D}$ and continued administration of vitamin D and calcium may mask the incidence of hypocalcemia or reduce its severity.

Another bone safety aspect that has been debated for several years is that prolonged use of bisphosphonates at high doses might be associated with micro fractures as described in animal models. ${ }^{54,55}$ These findings are not clear in the clinical setting as the doses used in these experiments are about 6-fold higher than that used in humans. Furthermore a study examining micro cracks in bones of dogs receiving alendronate and risedronate did not show effect on mechanical properties despite confirming increased micro-damage accumulation. ${ }^{56}$ Finally, in the FLEX trial, Recker et $\mathrm{al}^{57}$ did not find evidence of mineralization defect in bones of women who had been on alendronate for 10 years. Finally, no evidence associating ZOL and bone micro cracks has been reported.

\section{Clinical trials with zoledronic acid}

The effects of ZOL on bone mineral density (BMD) was investigated by Reid et al. ${ }^{50}$ They conducted a multinational one year study that randomized 45-80 year old postmenopausal women with $\mathrm{T}$-scores at the lumbar spine of less than $-2 \mathrm{SD}$ to receive either placebo or one of five ZOL regimens: $0.25 \mathrm{mg}, 0.5 \mathrm{mg}$, or $1 \mathrm{mg}$ at 3-month intervals; a single 4-mg dose; or 2 doses of $2 \mathrm{mg}$ administered 6 months apart. Although, the rate of increase tended to slow in the second half of the study, there were significant improvement in the average mean lumbar spine BMD and femoral neck BMD in the ZOL group compared with the placebo 
group (see Table 1). In contrast, BMD at the distal radius responded to ZOL treatment to a lesser extent. Significant decreases in bone turnover markers were also observed with the ZOL group. Markers of bone resorption reached a nadir at 1 month; however median decreases of $65 \%$ to $83 \%$ in serum C-telopeptide and $50 \%$ to $69 \%$ in the urinary N-telopeptide (Ntx):creatinine ratio were achieved. Furthermore the decrease in markers of resorption tended to be dose-dependent, particularly at 3 months of treatment. The incidence of adverse events was similar between all ZOL groups. The most commonly reported were myalgia and pyrexia.

Saag et $\mathrm{al}^{58}$ compared the onset of action, effects on bone resorption and tolerability of $\mathrm{ZOL}$ with alendronate. Postmenopausal women aged 45 to 79 years were randomized to receive either a single infusion of ZOL $5 \mathrm{mg}$ plus oral placebo or weekly oral alendronate $70 \mathrm{mg}$ plus a single placebo intravenous infusion. Women with either a femoral neck or lumbar spine BMD T-score of -2 or less at study initiation and no previous bisphosphonate use of 2 or more years were included.

At week 1, significantly lower mean urine NTx value was seen in the ZOL group as opposed to the alendronate group (15.2 $\mathrm{nmol}$ bone collagen equivalents (BCE)/mmol creatinine and $35.5 \mathrm{nmol} \mathrm{BCE} / \mathrm{mmol}$ creatinine, respectively). After this nadir, levels gradually increased but were significantly lower than the alendronate group throughout the 24-week study and remained stable within the study reference range of premenopausal women (17.8-46.4 nmol BCE/mmol creatinine) from week 12 to study end. Despite comparable adverse events $(91.3 \%$ in the ZOL group and $86.4 \%$ in the alendronate group) and similar premature discontinuation rates $(8.7 \%$ in the $\mathrm{ZOL}$ group and $8.5 \%$ in the alendronate group), the majority of patients preferred the annual ZOL infusion to the weekly alendronate, citing convenience as the primary factor.

In another multicenter trial, McClung et al ${ }^{49}$ similarly demonstrated better adherence and preference for annual infusion of ZOL over $70 \mathrm{mg}$ weekly of oral alendronate. Postmenopausal women who were receiving oral alendronate for at least 1 year and had lumbar spine or femoral neck BMD T-score values $\leq-2.0 \mathrm{SD}$ were randomized to one 15-min IV infusion of ZOL $5 \mathrm{mg}$ plus 52 weeks of oral placebo or one IV infusion of placebo plus 52 weeks of oral alendronate $70 \mathrm{mg}$. Bone marker levels decreased steadily in both groups and at month 12 remained in the lower half of the premenopausal reference range. The overall rates of adverse events were comparable in the 2 groups (ZOL $5 \mathrm{mg}, 86.7 \%$; alendronate $70 \mathrm{mg}, 80.4 \%)$. The majority $(78.7 \%$ ) of patients regardless of study groups expressed preference for once yearly infusion over weekly oral therapy. This result suggests that patients could be switched from oral alendronate to ZOL $5 \mathrm{mg}$ infusion without affecting the therapeutic benefits of oral bisphosphonates. This evidence is useful in cases in which oral bisphosphonates are not well tolerated or when adherence is a major issue.

Whether the actions of a single dose of ZOL on bone turnover and on BMD extends beyond 12 months was recently investigated by Borba et $\mathrm{al}^{59}$ This was a prospective study evaluating the changes in bone turnover at 12 months (T12) and 18 months (T18). The subjects had low BMD, had at least one other risk factor (in all but three) and had a mean age of $60.5 \pm 16$ years. Other metabolic bone diseases and past use of bisphosphonates were included in the exclusion criteria.

Median serum bone turnover markers levels were significantly suppressed at T12 and continued to be suppressed at T18 in comparison with baseline values. BMD at 12 months showed significant increase at the lumbar spine (LS) which was sustained at T18 (Table 1). A modest increase in total hip BMD was also evident and maintained at $\mathrm{T} 18$. The results of this study suggest that there may be a role of extending the time between infusions of ZOL beyond 12 months without losing efficacy and thereby possibly reducing costs and increasing adherence even further.

The long-term safety and efficacy of ZOL in women with postmenopausal osteoporosis was further evaluated by Devogelaer et al. ${ }^{60}$ This extension of a previous study ${ }^{50}$ was composed of 2 consecutive open-label, 2-year periods. Women who entered the first extension study received ZOL $0.5 \mathrm{mg}$ every 3 months; the others were administered ZOL $1 \mathrm{mg}$ every 3 months. Patients who entered the second extension study received either calcium only or ZOL $4 \mathrm{mg}$. Patients received treatment for either 2,3 , or 5 years. The efficacy of ZOL was measured by DEXA assessment of BMD at the proximal femur, lumbar spine, distal radius, and total body. BMD was found to increase in all three subgroups by the end of the 5-year total study period.

Arthralgia was the most commonly reported adverse event and 8 patients experienced serious adverse events, with 7 of the 15 total events reported as cardiovascular related. Interpretation of this study is however relatively limited due to the open-label design, small treatment groups, and lack of clarification of cardiovascular adverse events.

\section{Zoledronic acid and hip fractures}

Hip fractures are highly prevalent in older osteoporotic patients. However, despite a myriad of literature on 


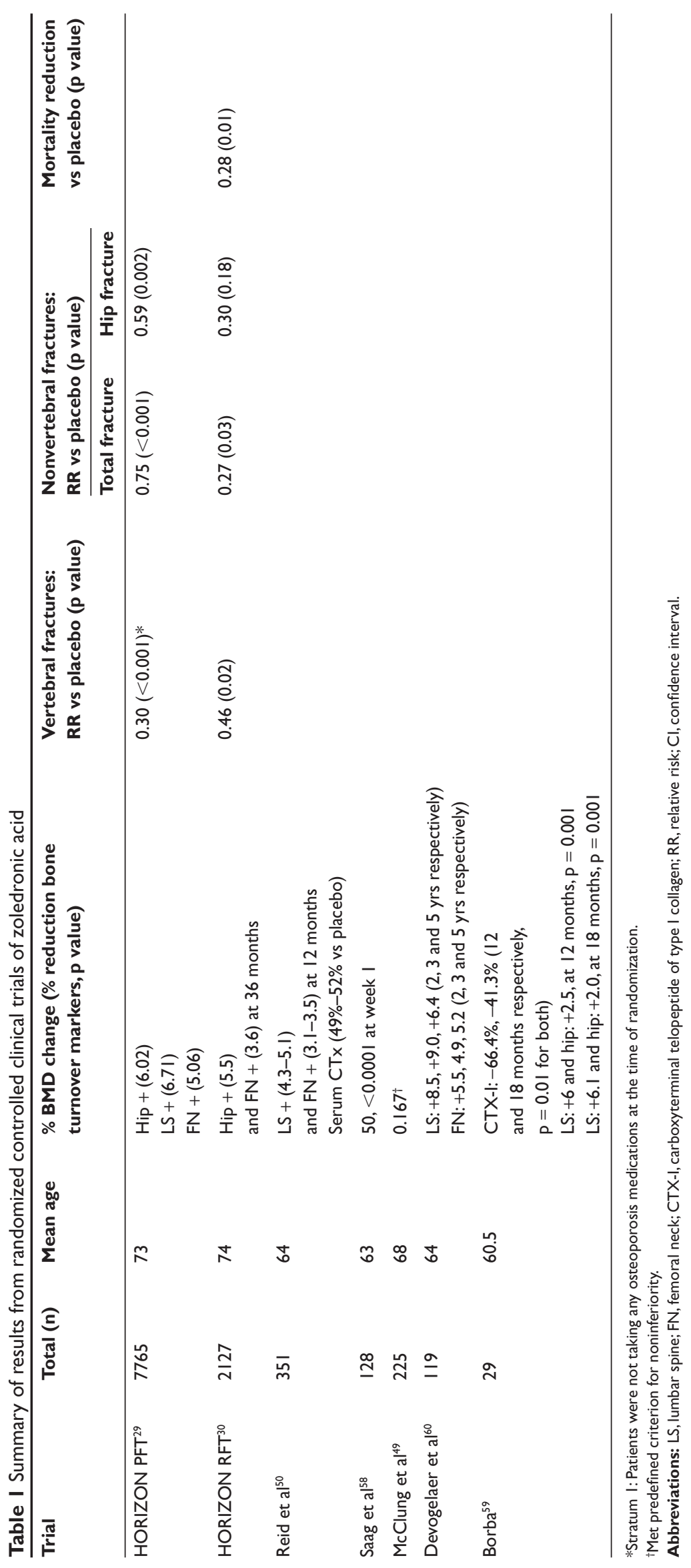


osteoporosis and its treatment, only a number of trials have assessed hip fractures as primary outcomes. ${ }^{61}$ In the case of ZOL, very few studies have been pursued looking at its effectiveness on primary and secondary prevention of hip fractures (Table 1) and just a few of them have looked specifically in older populations.

Recently, ZOL impact on re-fracture reduction was demonstrated in The HORIZON (Health Outcomes and Reduced Incidence with ZOL Once Yearly) Pivotal Fracture trial. ${ }^{29}$ This is the first trial to specifically evaluate the effects of ZOL on fractures. This double-blind, placebo-controlled trial enrolled 7765 postmenopausal women (mean age, 73 years) with confirmed osteoporosis and evidence of clinical fractures. Prior to randomization, patients were stratified according to their concomitant use of osteoporosis medications (raloxifene, calcitonin and hormone replacement therapy but not other bisphosphonates). 3889 were randomly assigned to receive a single 15-minute infusion of ZOL (5 mg) and 3876 were assigned to receive placebo at baseline, at 12 months, and at 24 months. The patients were followed for 3 years. All patients received oral daily calcium (1000-1500 mg) and vitamin D (400-1200 IU). Primary end points were new vertebral fractures (in patients not taking concomitant osteoporosis medications) and hip fractures (in all patients). Secondary end points included BMD, bone turnover markers, and safety outcomes.

In women not receiving concomitant medications, ZOL was found to reduce the risk of morphometric vertebral fracture by $70 \%$ during the 3 -year study period, as compared with placebo (3.3\% in the ZOL group vs $10.9 \%$ in the placebo group; absolute risk reduction of $7.6 \%$ ) and from both arms of treatment there was a reduction in the risk of hip fracture of $41 \%$ ( $1.4 \%$ in the ZOL group vs $2.5 \%$ in the placebo group; ARR 1.1\%) (Figure 2A).

Secondary outcomes also favored the ZOL group including a $33 \%$ reduction in any clinical fractures ( $8.4 \%$ vs $12.8 \%)$, $77 \%$ reduction in clinical vertebral fractures $(0.5 \%$ vs $2.6 \%)$

A)

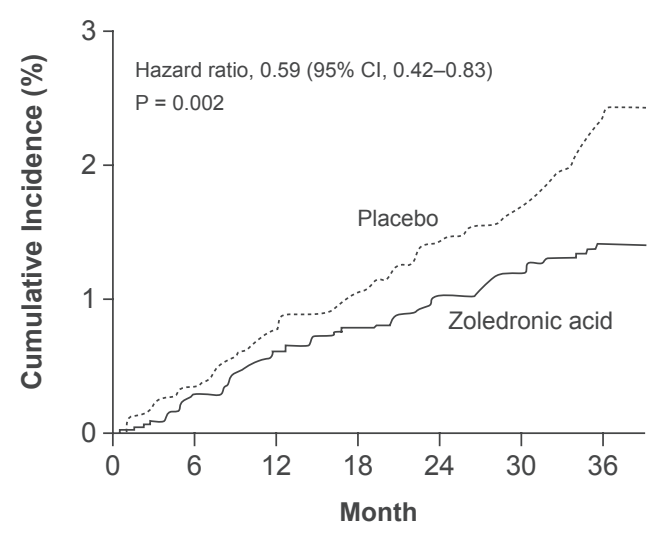

No. at Risk

$\begin{array}{llllllll}\text { Zoledronic acid } & 3875 & 3807 & 3674 & 3553 & 3494 & 3387 & 3161\end{array}$

$\begin{array}{llllllll}\text { Placebo } & 3861 & 3806 & 3694 & 3577 & 3499 & 3397 & 3144\end{array}$

B)

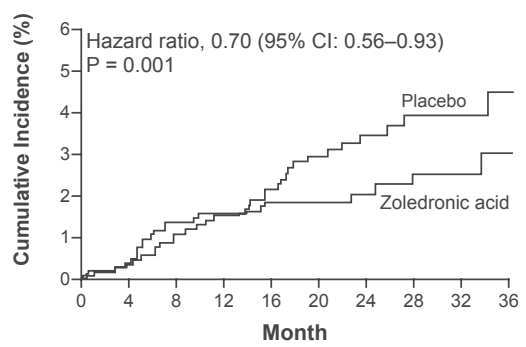

No. at Risk

Zoledronic acid $10651027978931794 \quad 664 \quad 499 \quad 344233 \quad 139$

$\begin{array}{lllllllllll}\text { Placebo } & 1062 & 1025 & 981 & 927 & 787 & 664 & 492 & 347 & 223 & 139\end{array}$
C)

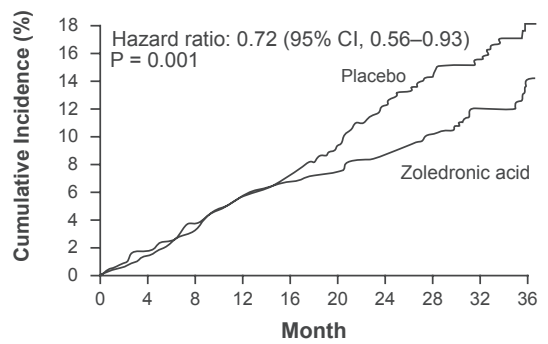

No. at Risk

Zoledronic acid1054 $1029987943 \quad 806 \quad 674507348 \quad 237 \quad 144$ $\begin{array}{lllllllllll}\text { Placebo } & 1057 & 1028 & 993 & 945 & 804 & 681 & 511 & 364 & 236 & 149\end{array}$

Figure 2 Clinical trials showing a significant reduction in the incidence of hip fractures in primary A) and secondary prevention B) In addition zoledronic acid a significant reduction in mortality after 2 years of treatment was found in the HORIZON trial. C) Adapted with permission (Part A) from Black D, Delmas P, Eastell R, et al. Once-yearly zoledronic acid for treatment of postmenopausal osteoporosis. N EnglJ Med. 2007;356(I8): 1809-1822; ${ }^{29}$ (Parts B and C) Lyles K, Colon-Emeric C, Magaziner J, et al Zoledronic acid and clinical features and mortality after hip fracture. N Engl J Med. 2007; 357(I8): 1799-1809. ${ }^{30}$ Copyright @ 2007 Massachusetts Medical Society.All rights reserved. 
and multiple vertebral fractures $(0.2 \%$ vs $2.3 \%)$ and $25 \%$ in nonvertebral fractures $(8.0 \%$ vs $10.7 \%)$. As compared with placebo, ZOL was also associated with a significant improvement in BMD and bone metabolism markers. BMD was increased at the total hip (6.02\%), lumbar spine (6.71\%) and the femoral neck $(5.06 \%)$. Tolerability was acceptable with $81 \%$ of patients receiving the maximum of three infusions. Retention rates were comparable with $2.1 \%$ of the ZOL group discontinuing treatment due to adverse events compared with $1.8 \%$ in the placebo group.

Adverse events, including change in renal function were similar in the two study groups. Interestingly, there was an inexplicable statistically significant increase in serious $\mathrm{AF}$ in the ZOL group compared with the placebo group (50 vs 20 patients, respectively), which occurred more than 30 days after infusion in 47 women in the treatment group. This suggests that it may not be related to the acute infusions. Hypocalcemia as a cause was deemed unlikely from the regular evaluation of electrolyte changes. The author reported that there were significant differences between study groups in the change in serum calcium from the level before the first infusion of ZOL to the level 9 to 11 days after the infusion, but the magnitude of the difference was relatively small (a reduction of $0.2 \pm 0.5 \mathrm{mg} / \mathrm{dL}$ in the ZOL group vs an increase of $0.03 \pm 0.4$ in the placebo group). This difference was not evident at 12 months, and no significant changes occurred with subsequent doses. Furthermore, the change in calcium levels after the first infusion did not differ between women with AF and those without AF. Over the 3 years of the study, mean serum calcium levels increased in both study groups, although the mean increase was slightly larger in the placebo group than in the ZOL group. Magnesium and potassium levels did not differ between the two study groups and were similar in women with and those without AF. It should be noted also that episodes of AF did not cluster in time immediately after any infusion, when serum electrolytes are most affected. Taken together, these findings suggest a possible alternative explanation.

Finally, there are some features of this study that make it unique in terms of the population studied. Although an active comparator would be of great clinical interest, the numbers required for such a trial to demonstrate equivalent or superior fracture risk reductions would be difficult to obtain and possibly prohibitive. Instead, to simulate reality, the protocol specified that patients had to be unable or unwilling to take oral bisphosphonates and all women were counseled on the risk of fracture and the availability of approved osteoporosis medications. Another allowance that mirrors clinical reality was that women who were receiving nonbisphosphonate treatments for osteoporosis (including hormone therapy, raloxifene, and calcitonin) were included ("stratum 2"), and all patients were free to begin any of these treatments during the study while continuing to receive the study treatment. Furthermore, the researchers monitored patients for excessive bone loss or multiple fractures, and women who met either criterion were again counseled about alternative treatment options.

Nevertheless, the evidence provided by this study suggests that a once-yearly infusion of ZOL during a 3-year period significantly reduced the risk of hip fractures in this particular population.

\section{Zoledronic acid and mortality}

The second major trial is the HORIZON recurrent fracture trial. ${ }^{30}$ This well designed trial in a population of frail older subjects constitutes the first trial of an osteoporosis intervention to show a reduction in mortality. This 3-year, multicenter, multinational, double-blind, placebo-controlled trial of 2127 patients with a recent hip fracture assessed the effect of ZOL administered within 90 days after surgical repair. Men and women 50 years of age or older were eligible for inclusion within 90 days after surgical repair of a hip fracture sustained with minimal trauma (ie, a fall from standing height or a lower height). All patients who were enrolled in the trial had undergone repair of a hip fracture and were unable or unwilling to take an oral bisphosphonate. Additional enrollment criteria included being ambulatory before the hip fracture and having both legs. Concomitant therapy with nasal calcitonin, selective estrogen-receptor modulators, hormone replacement, tibolone, and external hip protectors was allowed at the discretion of the investigator. Previous use of bisphosphonates or parathyroid hormone was allowed after a washout period that varied according to the drug and the duration of its use. Previous use of strontium or sodium fluoride was not allowed. Any patient with a serum 25-hydroxyvitamin D level of $15 \mathrm{ng} / \mathrm{mL}$ or less, or if the level was not available, a loading dose of either vitamin D3 or D2 (at a dose of 50,000 to $125,000 \mathrm{IU}$ ) was given orally or intramuscularly 14 days before the first infusion of ZOL. Thereafter, all patients received daily supplementation with oral calcium (1000 to $1500 \mathrm{mg}$ ) and vitamin D (800 to $1200 \mathrm{IU})$. Exclusion criteria were previous hypersensitivity to a bisphosphonate, a potential for pregnancy, a calculated creatinine clearance of less than $30 \mathrm{~mL}$ per minute, a corrected serum calcium level of more than $11.0 \mathrm{mg} / \mathrm{dL}(2.8 \mathrm{mmol} / \mathrm{L})$ or less than $8.0 \mathrm{mg} / \mathrm{dL}$ ( $2.0 \mathrm{mmol} / \mathrm{L})$, active cancer, metabolic bone disease other than osteoporosis, and a life expectancy of less than 6 months in the investigator's judgment. BMD 
at the hip and femoral neck and the calculated creatinine clearance were determined at baseline and annually. The primary endpoint was the incidence of further hip fractures and secondary endpoints included the change in BMD in the nonfractured hip, new vertebral fractures, and pre-specified safety endpoints, including death.

Of a total of 2127 patients, 1065 patients were randomly assigned to receive ZOL, and 1062 patients were assigned to receive placebo; $71.3 \%$ of the patients completed the trial. The median follow-up time was 1.9 years. Baseline demographic and clinical characteristics were similar in the two groups, with $41.8 \%$ of patients having a $\mathrm{T}$ score less than $-2.5 \mathrm{SD}$ at the femoral neck.

At 24 months ZOL was associated with a rate of new clinical fractures of $8.6 \%$, as compared with $13.9 \%$ in the placebo group, an absolute risk reduction of $5.3 \%$ and a relative reduction of $35 \%$. Among patients who had a fracture, the mean time to clinical fracture was 39.8 months in the ZOL group and 36.4 months in the placebo group. The risk reduction was very similar in the intention-to-treat and per-protocol populations. The rates of new clinical vertebral and nonvertebral fractures were significantly lower (1.7\% vs $3.8 \%$ and $7.6 \%$ vs $10.7 \%$, respectively).

However the incidence of new hip fractures was not significantly different $(2.0 \%$ vs $3.5 \%$, a nonsignificant reduction in relative risk of $30 \%$ ) (Figure $2 \mathrm{~B}$ ). Although in a post hoc analysis, significant divergence in the fracturefree survival curves between the two groups for all clinical fractures was seen as early as 12 months ( $p=0.02$ by the logrank test). BMD at the total hip increased in the ZOL group by $2.6 \%, 4.7 \%, 5.5 \%$ at 12,24 and 36 months respectively compared with a decline BMD in the placebo group by $1.0 \%$, $0.7 \%$, and $0.9 \%$, respectively. BMD at the femoral neck also increased in the ZOL group by $0.8 \%, 2.2 \%$ and $3.6 \%$ at 12,24 and 36 months respectively and declined in the placebo group by $1.7 \%, 2.1 \%$, and $0.7 \%$, respectively over the same period. All increases in BMD were statistically significant compared with placebo. Finally, the difference in delayed union of fractured bone between the two study groups was not significant $(3.2 \%$ in the ZOL group and vs $2.7 \%$ in the placebo group; 95\% CI, 0.72 to $1.90 ; \mathrm{p}=0.61$ ).

In the safety analysis, mortality rates were similar in the first 12 months but were significantly different at 24 months with $9.6 \%$ in the ZOL group vs $13.3 \%$ in the placebo group (a relative reduction of $28 \%$ in the risk of death) (Figure $2 \mathrm{C}$ ). The causes of deaths were primarily from cardiovascular and cerebrovascular diseases. Serious adverse events occurred with similar frequency in the two groups $(38.3 \%$ in the ZOL group and $41.2 \%$ in the placebo group). More patients in the ZOL group than in the placebo group reported pyrexia, myalgia, bone pain, and musculoskeletal pain. More patients in the placebo group (11.4\%) reported having fallen than in the ZOL group (9.7\%). The incidence of cardiovascular events was similar in the two groups. A total of 24 patients in the ZOL group (2.3\%) and 39 patients in the placebo group (3.7\%) had a serious adverse event of arrhythmia; rates of AF were similar in the two groups $1.1 \%$ vs $1.3 \%$. The incidence of renal adverse events was similar in the two study groups, including events among patients with a baseline creatinine clearance of 30 to $60 \mathrm{~mL}$ per minute.

Although no specific case-finding effort was made, no cases of ONJ were reported. In summary, this is a well conducted trial showing that treatment efficacy is possible in the frail elderly group in whom oral bisphosphonates is less efficacious due to side effects and noncompliance. In addition, the reduction in number of deaths is almost the same as the reduction in number of fractures suggesting that fewer post fracture deaths are unlikely to account for the benefit seen. ${ }^{62}$ Whether concomitant treatment with calcitonin, selective estrogen-receptor modulators, and hormone-replacement therapy had an effect on the results is also not certain. However when this study was designed, the only therapies that had been proven to reduce the risk of hip fractures were alendronate and risedronate. ${ }^{63-65}$ Thus, it is likely that the use of nonbisphosphonate therapies did not have an important influence on the overall outcome. In addition the study by Black et $\mathrm{al}^{29}$ did not show any difference in the incidence of hip, nonvertebral, or all clinical fractures between subjects who received, and those who did not receive, concomitant therapy (10\% in both study groups).

For the mortality results, it is more likely that the reason for this reduction in mortality is multifactorial ${ }^{62}$ as suggested by the separation of the mortality curves occurring after a year; however, further investigation and more detailed analysis of these data may be needed to elucidate the reasons. It is also interesting that despite the reduction in hip fracture not reaching statistical significance the number needed to treat of 67 to 71 is quite comparable to other bisphosphonate trials. ${ }^{29,63}$ The baseline risk of death in this patient population was substantially different from that in previous ZOL trials ${ }^{29}$ and may also account for some of the variance in results. In addition, the study was not powered to show the differences between treatment groups for this end point.

Special comments should also be made regarding the incidence of AF. Although an increased rate of AF in association with this medication had been a concern in the HORIZON 
trial, ${ }^{29}$ it is interesting that in this study, the incidence did not differ between the medication and placebo groups.

\section{Zoledronic acid in the older population}

Although the evidence supporting ZOL as a choice for the prevention of osteoporosis fractures in older patients is scarce, there are several characteristics of ZOL that make it an appealing choice in this population.

In terms of compliance, several studies have demonstrated that older patients are more prone to discontinue their treatment after 6 months of administration due to several reasons including difficulties with the strict instructions required for oral bisphosphonates, the lack of symptoms associated with osteoporosis and the poor encouragement received from their family practitioners to continue with their treatment. ${ }^{66-68}$ In this situation, ZOL constitutes an important choice since it only requires 1 dose every year. Nevertheless, the patients should continue taking vitamin $\mathrm{D}$ and calcium in a permanent manner.

A second argument in favor of ZOL in older patients is the recent evidence showing that additionally to its effect on re-fracturing, patients receiving ZOL showed lower mortality rates. ${ }^{30}$ This is a particularly important effect especially in the older and frail populations. However, the mechanisms that explain this effect remain unknown.

Finally, no studies have been pursued in long-term institutions looking at the effectiveness and cost-benefit of ZOL in institutionalized elderly patients. For patients that are in long-term care institutions, the population at higher risk of fractures according to the literature, ${ }^{18,69} \mathrm{ZOL}$ could become an important choice for fracture prevention.

\section{Conclusion}

Hip fractures are an increasing global problem as the population ages. The human costs and economic burden will escalate proportionately. Pharmacotherapy to reduce the morbidity and mortality associated with hip fractures is effective and proven although is still limited by convenience, tolerability and thus compliance. Decisions regarding which agent should be based on these factors and sound hip fracture data. Only alendronate, ${ }^{70}$ risedronate $^{71}$ and $\mathrm{ZOL}^{29,30}$ have been proven to reduce nonvertebral and in particularly hip fractures in the intention to treat populations from more than one trial and randomized controlled trials of at least 3 years' duration. ${ }^{65} \mathrm{ZOL}$ offers advantages of convenience, tolerability and thus greater compliance. The findings that $\mathrm{ZOL}$ reduces the recurrence of fractures in patients who already sustained a hip fracture and increase survival, means that there is now available therapy for a population that was largely without therapy before these recent trials were pursued.

\section{Acknowledgments}

Dr Duque holds a Fellowship from the University of Sydney Medical Research Foundation. Dr Demontiero has a postdoctoral scholarship in aging research from the Rebecca $\mathrm{L}$ Cooper Medical Research Foundation.

\section{Disclosures}

The authors disclose no conflicts of interest.

\section{References}

1. Raisz L. Pathogenesis of osteoporosis: concepts, conflicts, and prospects. J Clin Invest. 2005;115(12):3318-3325.

2. Clowes J, Eastell R. The role of bone turnover markers and risk factors in the assessment of osteoporosis and fracture risk. Baillieres Best Pract Res Clin Endocrinol Metab. 2000;14(2):213-232.

3. Burge R, Dawson-Hughes B, Solomon D, Wong J, King A, Tosteson A. Incidence and economic burden of osteoporosis-related fractures in the United States, 2005-2025. J Bone Miner Res. 2007;22(3):465-475.

4. Siris E, Miller P, Barrett-Connor E, et al. Identification and Fracture Outcomes of Undiagnosed Low Bone Mineral Density in Postmenopausal Women . Results From the National Osteoporosis Risk Assessment. JAMA. 2001;286(22):2815-2822.

5. Wilkins C, Birge S. Prevention of osteoporotic fractures in the elderly. Am J Med. 2005;118(11):1190-1195.

6. Reginster J, Burlet N. Osteoporosis: A still increasing prevalence. Bone. 2006;38(2 Suppl 1):S4-S9

7. Cole Z, Dennison E, Cooper C. Osteoporosis epidemiology update. Curr Rheumatol Rep. 2008;10(2):92-96.

8. Johnell O, Kanis J. Epidemiology of osteoporotic fractures. Osteoporos Int. 2005;16 Supp1 2:S3-S7.

9. Cooper C, Atkinson E, Jacobsen S, O'Fallon W, Melton Lr. Populationbased study of survival after osteoporotic fractures. Am J Epidemiol. 1993;137(9):1001-1005.

10. Farahmand B, Michaelsson K, Ahlbom A, Ljunghall S, Baron J. Survival after hip fracture. Osteoporos Int. 2005;16:1583-1590.

11. Haentjens P, Boonen S. Excess mortality after hip fracture among postmenopausal women and ageing men: evidence from data searches and life-table analyses for gender-related differences in absolute risk of death after hip fracture. Osteporosis Int. 2007;18 Suppl 1:S5-S6.

12. Torgerson D, Dolan P. Prescribing by general practitioners after an osteoporotic fracture. Ann Rheum Dis. 1998;57(6):378-379.

13. Kamel H, Hussain M, Tariq S, Perry H, Morley J. Failure to diagnose and treat osteoporosis in elderly patients hospitalized with hip fracture. Am J Med. 2000;109(4):326-328.

14. Kamel H, Duthie E. The underuse of therapy in the secondary prevention of hip fractures. Drugs Aging. 2002;19(1):1-10.

15. Andrade S, Majumdar S, Chan K, et al. Low frequency of treatment of osteoporosis among postmenopausal women following a fracture. Arch Intern Med. 2003;163(17):2052-2057.

16. Solomon D, Finkelstein J, Katz J, Mogun H, Avorn J. Underuse of osteoporosis medications in elderly patients with fractures. Am J Med. 2003;115(5):398-400.

17. Colón-Emeric C, Lyles K, House P, et al. Randomized trial to improve fracture prevention in nursing home residents. Am J Med. 2007;120(10):886-892. 
18. Klotzbuecher C, Ross P, Landsman P, Abbott Tr, Berger M. Patients with prior fractures have an increased risk of future fractures:a summary of the literature and statistical synthesis. J Bone Miner Res. 2000;15(4):721-739.

19. Kanis J, Johnell O, Laet CD, et al. A meta-analysis of previous fracture and subsequent fracture risk. Bone. 2004;35(2):375-382.

20. Center J, Bliuc D, Nguyen T, Eisman J. Risk of subsequent fracture after low-trauma fracture in men and women. JAMA. 2007;297:387-394.

21. Friesendorff MV, Besjakov J, Åkesson K. Long-term survival and fracture risk after hip fracture: a 22-year follow-up in women. $J$ Bone Miner Res. 2008;23(11):1832-1841.

22. Hamlet W, Lieberman J, Freedman E, Dorey F, Fletcher A, Johnson E. Influence of health status and the timing of surgery on mortality in hip fracture patients. Am J Orthop. 1997;26(9):621-627.

23. Center J, Nguyen T, Schneider D, Sambrook P, Eisman J. Mortality after all major types of osteoporotic fracture in men and women: an observational study. Lancet. 1999;353(9156):878-882.

24. Bhattacharyya T, Iorio R, Healy W. Rate of and risk factors for acute inpatient mortality after orthopaedic surgery. J Bone Joint Surg Am. 2002;84-A(4):562-572.

25. Magaziner J, Fredman L, Hawkes W, et al. Changes in functional status attributable to hip fracture: a comparison of hip fracture patients to community-dwelling aged. Am J Epidemiol. 2003;157(11):1023-1031.

26. Russell R. Determinants of structure-function relationships among bisphosphonates. Bone 2007;40(5):S21-S25.

27. Shoback D. Update in osteoporosis and metabolic bone disorders. J Clin Endocrinol Metab. 2007;92(3):747-753.

28. Boonen S, Vanderschueren D, Venken K, Milisen K, Delforge M, Haentjens P. Recent developments in the management of postmenopausal osteoporosis with bisphosphonates: enhanced efficacy by enhanced compliance. J Intern Med. 2008;264(4):315-332.

29. Black D, Delmas P, Eastell R, et al. Once-yearly zoledronic acid for treatment of postmenopausal osteoporosis. $N$ Engl J Med. 2007;356(18):1809-1822.

30. Lyles K, Colon-Emeric C, Magaziner J, et al. Zoledronic acid and clinical features and mortality after hip fracture. $N \mathrm{Engl} \mathrm{J} \mathrm{Med.}$ 2007;357(18):1799-1809.

31. Dunford J, Thompson K, FP FC, et al. Structure-activity relationships for inhibition of farnesyl disphosphate synthase in vitro and inhibition of bone resorption in vivo by nitrogen-containing bisphosphonates. $J$ Pharmacol Exp Ther. 2001;296(2):235-242.

32. Rodan G, Fleisch H. Bisphosphonates: mechanism of actions. J Clin Invest. 1996;97:2692-2696.

33. Lehenkari P, Kellinsalmi M, Näpänkangas J, et al. Further insight into mechanism of action of clodronate:inhibition of mitochondrial ADP/ATP translocase by a nonhydrolyzable, adenosine containing metabolite. Mol Pharmacol. 2002;61(5):1255-1262.

34. Frith J, Monkkonen J, Auriola S, Mönkkönen H, Rogers M. The molecular mechanism of action of the antiresorptive and antiinflammatory drug clodronate: evidence for the formation in vivo of a metabolite that inhibits bone resorption and causes osteoclast and macrophage apoptosis. Arthritis Rheum. 2001;44(9):2201-2210.

35. Russell R. Bisphosphonates: from bench to bedside. Ann N Y Acad Sci. 2006;1068:367-401

36. Bergstrom J, Bostedor R, Masarachia P, Reszka A, Rodan G. Alendronate is a specific, nanomolar inhibitor of farnesyl diphosphate synthase. Arch Biochem Biophys. 2000;373(1):231-241

37. Li E, Davis L. Zoledronic acid: a new parenteral bisphosphonate. Clin Ther. 2003;25(11):2669-2708.

38. Green J. Chemical and biological prerequisites for novel bisphosphonate molecules: Results of comparative preclinical studies. Semin Oncol. 2001;28(2 Suppl 6):4-10.

39. Benford H, McGowan N, Helfrich M, Nuttall M, Rogers M. Visualization of bisphosphonateinduced caspase-3 activity in apoptotic osteoclasts in vitro. Bone. 2001;28(5):465-473.

40. Boissier S, Ferreras M, Peyruchaud O, et al. Bisphosphonates inhibit breast and prostate carcinoma cell invasion, an early event in the formation of bone metastases. Cancer Res. 2000;60(11):2949-2954
41. Nancollas G, Tang R, Guide S, et al. Mineral binding affinities and zeta potentials of bisphosphonates. J Bone Miner Res. 2002; 17 Suppl 1:S368.

42. Nancollas G, Tang R, Phipps R, et al. Novel insights into actions of bisphosphonates on bone: differences in interactions with hydroxyappetite. Bone. 2006;38(5):617-627.

43. Hornby S, Evans G, Hornby S, Pataki A, Glatt M, Green J. Long-term zoledronic acid treatment increases bone structure and mechanical strength of long bones of ovariectomized adult rats. Calcif Tissue Int. 2003;72(4):519-527.

44. Binkley N, Kimmel D, Bruner J, et al. Zoledronate prevents the development of absolute osteopenia following ovariectomy in adult rhesus monkeys. J Bone Miner Res. 1998;13(11):1775-1782.

45. Novartis, Reclast (zoledronic acid). East Hanover, NJ August 2007.

46. Green J, Muller K, Jaeggi K. Preclinical pharmacology of CGP 42'446, a new, potent, heterocyclic bisphosphonate compound. J Bone Miner Res. 1994;5(9):745-751.

47. Chen T, Berenson J, Vescio R, et al. Pharmacokinetics and pharmacodynamics of zoledronic acid in cancer patients with bone metastases. J Clin Pharmacol. 2002;42(11):1228-1236

48. Boonen S, Sellmeyer D, Lippuner K, et al. Renal safety of annual zoledronic acid infusions in osteoporotic postmenopausal women. Kidney Int. 2008;74:641-648.

49. McClung M, Recker R, Miller P, et al. Intravenous zoledronic acid $5 \mathrm{mg}$ in the treatment of postmenopausal women with low bone density previously treated with alendronate. Bone. 2007;41(1):122-128.

50. Reid I, Brown J, Burckhardt P, et al. Intravenous Zoledronic acid in postmenopausal women with low bone mineral density. $N$ Engl J Med. 2002;346(9):653-661.

51. Patrick M, Purcell WB, Boyd IW. Bisphosphonates and osteonecrosis of the jaw. MJA. 2005;182(8):417-418.

52. Vieillard M, Maes J, Penel G, et al. Thirteen cases of jaw osteonecrosis in patients on bisphosphonate therapy. Joint, Bone, Spine: Revue du Rhumatisme. 2008;75(1):34-40.

53. Brooks J, Gilson A, Sindler A, Ashman S, Schwartz K, Nikitakis N. Osteonecrosis of the jaws associated with use of risedronate: report of 2 new cases. Oral Surg Oral Med Oral Pathol Oral Radiol Endod. 2007;103(6):780-786

54. Mashiba T, Mori S, Burr D, et al. The effects of suppressed bone remodeling by bisphosphonates on microdamage accumulation and degree of mineralization in the cortical bone of dog rib. J Bone Miner Metab. 2005;23 Suppl:S36-S42.

55. Komatsubara S, Mori S, Mashiba T, et al. Long term treatment of incdronate disodium accumulates microdamage but improves the trabecular bone microarchitecture in dog vertebra. J Bone Miner Res. 2003; 18:512-520.

56. Allen M, Iwata K, Phipps R, others a. Residroante and alendroante similarly suppress remodeling and increase microdamage in beagles after 1 year of treatment at clinical doses. 27th Annual Meeting of the American Society for Bone and Mineral Research. Nashville, Tennessee, USA: J Bone Miner Res. 2005:S22.

57. Recker R, Ensrud K, Diem S, et al. Normal Bone Histomorphometry and $3 \mathrm{D}$ microarchitecture after 10 years alendronate treatment of postmenopausal women. 26th Annual meeting of the American Society for Bone and Mineral Research. Seattle, Washington, USA. 2004:S45.

58. Saag K, Lindsay R, Kriegman A, Beamer E, Zhou W. A single zoledronic acid infusion reduces bone resorption markers more rapidly than weekly oral alendronate in postmenopausal women with low bone mineral density. Bone. 2007;40(5):1238-1243.

59. Borba V, Paz-Filho G. Bone turnover 18 months after a single intravenous dose of zoledronic acid. Int J Clin Pract. 2007;61(6):1058-1062.

60. Devogelaer J, Brown J, Burkhardt P, et al. Zoledronic acid efficacy and safety over five years in postmenopausal osteoporosis. Osteoporos Int. 2007;18(9):1211-1218.

61. Bruyere O, Brandi M, Burlet N, et al. Post-fracture management of patients with hip fracture: a perspective. Curr Med Res Opin. 2008;24(10):2841-2851. 
62. Duque G. Intravenous zoledronic acid reduced new clinical fractures and deaths in patients who had recent surgery for hip fracture. ACP J Club. 2008;148(2):40.

63. Black D, Cummings S, Karpf D, et al. Randomised trial of effect of alendronate on risk of fracture in women with existing vertebral fractures. Lancet. 1996;348(9041):1535-1541.

64. Cranney A, Waldegger L, Zytaruk N, et al. Risedronate for the prevention and treatment of postmenopausal osteoporosis. Cochrane Database Syst Rev. 2003(4):CD004523.

65. Duque G, Demontiero O, Troen B. The prevention and treatment of senile osteoporosis and hip fractures. Minerva Medica (in press).

66. Silverman S. Adherence to medications for the treatment of osteoporosis. Rheum Dis Clin North Am. 2006;32(4):721-731.

67. Yood R, Emani S, Reed J, Lewis B, Charpentier M, Lydick E. Compliance with pharmacologic therapy for osteoporosis. Osteoporos Int. 2003;14(12):965-968.
68. Siris E, Harris S, Rosen C, et al. Adherence to bisphosphonate therapy and fracture rates in osteoporotic women: relationship to vertebral and nonvertebral fractures from 2 US claims databases. Mayo Clin Proc. 2006;81(8):1013-1022.

69. Colón-Emeric C, Lyles K, Levine D, et al. Prevalence and predictors of osteoporosis treatment in nursing home residents with known osteoporosis or recent fracture. Osteoporos Int. 2007;18(4):553-559.

70. Wells G, Cranney A, Peterson J, et al. Alendronate for the primary and secondary prevention of osteoporotic fractures in postmenopausal women. Cochrane Database Syst Rev. 2008(Issue 1. Art. No.: CD001155).

71. Wells G, Cranney A, Peterson J, et al. Risedronate for the primary and secondary prevention of osteoporotic fractures in postmenopausal women. Cochrane Database Syst Rev. 2008;(1):CD004523. 